\title{
Joint rate control and power allocation for low-latency reliable D2D-based relay network
}

\author{
Yahui Wang ${ }^{1}$, Yanhua He ${ }^{1}$, Chen Xu ${ }^{1}$ Zhenyu Zhou ${ }^{*}$ (D), Shahid Mumtaz ${ }^{2}$, Jonathan Rodriguez 2,3 \\ and Haris Pervaiz ${ }^{4}$
}

\begin{abstract}
Emerging 5G applications impose stringent requirements on network latency and reliability. In this work, we propose a low-latency reliable device-to-device (D2D) relay network framework to improve the cell coverage and user satisfaction. Particularly, we develop a cross-layer low-complexity resource allocation algorithm, which jointly optimizes the rate control and power allocation from a long-term perspective. The long-term optimization problem is transformed into a series of short-term subproblems by using Lyapunov optimization, and the objective function is separated into two independent subproblems related to rate control in network layer and power allocation in physical layer. Next, the Karush-Kuhn-Tucher (KKT) conditions and alternating direction method of multipliers (ADMM) algorithm are employed to solve the rate control subproblem and power allocation subproblem, respectively. Finally, simulation results demonstrate that the proposed algorithm can reach $99.9 \%$ of the optimal satisfaction of D2D pairs with lower average network delay compared to the baseline algorithm. Furthermore, the convergence time of the ADMM-based power allocation algorithm is only about $1.7 \%$ of that by using the CVX toolbox.
\end{abstract}

Keywords: D2D-based relay network, Rate control, Power allocation, Lyapunov optimization, ADMM algorithm, Low-latency and reliability

\section{Introduction}

With the explosive growth of mobile applications, it is predicted that approximately 50 billion devices will interconnect to the network by 2020 [1,2]. Cell-edge devices are likely to experience poor quality of service (QoS) and quality of experience (QoE) due to the long distance and time-varying channel states between devices and the base station (BS). Device-to-device (D2D)-based relay communication, as a key technology of 5G, can improve data transmission and network coverage by assisting users with inferior channel conditions via multi-hop transmissions. Specifically, the transmitters (TXs) of D2D pairs can act as relay nodes to reduce the transmission distance and conditions. improve the channel The required data of D2D receivers (RXs) is transmitted from the BS to

\footnotetext{
*Correspondence: zhenyu_zhou@ncepu.edu.cn

${ }^{1}$ The State Key Laboratory of Alternate Electrical Power System with

Renewable Energy Sources, School of Electrical and Electronic Engineering,

North China Electric Power University, Beijing, China

Full list of author information is available at the end of the article
}

the nearby TXs, which is stored in the queue buffers of TXs before being transmitted to RXs. Compared to conventional multi-hop relay network, the D2D-based relay network can be deployed underlying conventional cellular networks, which enables centralized resource management and coordination.

However, despite the advantages described above, the widespread deployment of D2D-based relay network still faces some challenges.

Firstly, there lacks a cross-layer resource allocation scheme to guarantee the reliability of network operation as well as to satisfy the low-latency requirements of applications. Since both the arrival rate of required data at TXs and channel conditions among D2D pairs are varying over time, there requires a joint optimization of the rate control in network layer and transmission rate in physical layer. Traditional schemes, which only consider the optimization of the physical-layer transmission rate while ignoring the arrival rate in network layer, will result in the data imbalance between the arrival rate 
and the transmission rate. The data imbalance will cause queue backlog and packet drop at TXs due to the limited queue buffering capability of TXs, which leads to intolerable latency and network unreliability.

Second, there lacks an effective online resource allocation scheme which optimizes the network performance from a long-term perspective. Conventional short-term optimization cannot satisfy the long-term optimization objective and constraints, which will lead to severe performance degradation since resources are only allocated based on instantaneous states and constraints. In addition, it is difficult to obtain accurate future information in practical applications due to the casuality constraint.

Last but not least, the computational complexity of traditional resource allocation algorithms increase dynamically as the number of D2D pairs increases. The reason is that numerous optimization variables in the network are coupled with each other, e.g., the constraint of sum rate and sum power consumption, which leads to prohibitive computational complexity. On the other hand, the optimization problem with coupled constraints is carried out in a time slot basis, which further increases computational complexity.

In this paper, to solve the abovementioned challenges, we propose a cross-layer online joint resource allocation algorithm to optimize the long-term satisfaction of D2D pairs while maintaining network reliability and reduce transmission delay. The main contributions of this work are summarized as follows:

1. We transform the long-term joint rate control and power allocation problem into a series of short-term optimization problems by using Lyapunov optimization [3]. At each time slot, the cross-layer joint optimization problem can be decomposed into two separate subproblems and solved independently. The proposed scheme guarantees a $\left[\mathcal{O}\left(\frac{1}{V}\right), \mathcal{O}(V)\right]$ tradeoff between queue stability and D2D pair satisfaction.

2. The power allocation problem has high computational complexity due to the coupling of optimization variables among different $\mathrm{D} 2 \mathrm{D}$ pairs. To provide a tractable solution, we developed an alternating direction method of multipliers (ADMM)-based low-complexity power allocation scheme, which decomposes the large problem into a series of smaller subproblems and coordinates the solutions of these subproblems to find the solution of the original problem.

3. Simulation results demonstrate that the proposed joint optimization scheme can converge quickly and approximate the optimal solution. Moreover, we analyze the tradeoff between satisfaction of D2D pairs and network delay, which proves that significant performance can be improved by the proposed algorithm.

The remaining parts of this paper are organized as follows. Section 2 describes the related works. Section 3.1 introduces the power allocation model, the queue backlog model and satisfaction model. The problem formulation is presented in Section 3.2. The online joint rate control and power allocation algorithm based on Lyapunov optimization and ADMM and the performance analysis of the proposed algorithm are described in Sections 3.3 and 3.4, respectively. Simulation results are presented in Section 4 . The conclusion and future works are summarized in Section 5.

\section{Related works}

Due to the advantages of D2D-based relay network such as enlarging cell coverage, reducing network delay and enhancing network reliability, it has aroused widespread concern in both academia and industry. A multidimensional optimization algorithm was proposed to solve a content distribution problem in multi-hop D2D relay networks [4], which can effectively reduce the average delay in the network. In [5], Zhou et al. studied the D2D communication underlying cellular networks and proposed a joint channel selection and power allocation optimization algorithm to improve the energy efficiency subject to various QoS constraints. In [6], Dang et al. proposed a full-duplex based D2D multi-hop communication framework, where the data forwarded between D2D transmitters and receivers are assisted by using multiple relays. However, these works mainly focus on the optimization of short-term network performance, e.g., instantaneous network capacity, energy efficiency, and transmission latency, while ignoring the optimization of time-average performance.

Lyapunov optimization is a powerful methodology for studying long-term optimization problems, which is able to transform long-term objective function into a series of short-term subproblems and transform the long-term constraints into queue stability constraints. It has been applied in various application scenarios such as D2D networks [7], edge computing [8], and OFDMA-based cellular networks [9]. In [3], Sheng et al. proposed a resource allocation algorithm to maximize the energy efficiency of D2D communication underlaying cellular networks subject to the time-average and network stability constraints by combining fractional programming and Lyapunov optimization. In [10], Guo et al. proposed a cross-layer joint rate control and resource allocation scheme, which can maximize the time-average user satisfaction based on Lyapunov optimization. In [11], Peng et al. considered the energy efficiency optimization problem in multimedia 
HCRANs subject to individual front-haul capacity as well as multiple interference constraints to sense queue and proposed an online resource allocation algorithm based on Lyapunov optimization. However, when optimizing the performance of the overall network, the computational complexity increases dramatically with the number of devices due to the coupling of optimization variables and constraints across devices.

ADMM algorithm can solve some specific convex optimization problems with a much lower complexity, because both the primal and dual variables are updated in an alternative direction to increase the convergence speed [12]. It employs a decomposition-coordination procedure, in which the global optimization problem is firstly decomposed into numerous small subproblems, and then the solutions to these subproblems are calculated, updated, and coordinated to find a solution to the global problem. ADMM has been widely adopted in addressing large-scale optimization problems in various application scenarios. In [13], Li et al. decoupled the power constraint and objective function by employing ADMM and proposed a robust design of transceiver multi-cell distributed antenna network with numerous remote radio heads. In [14], Ling et al. proposed a weighted ADMM algorithm to solve the consensus optimization problem in decentralized networks, which is able to minimize the communication cost of optimization. In [15], Chen et al. combined the ADMM algorithm with the convex-concave procedure to reduce the complexity and improve system performance for large-scale multi-group multicast beamforming problems.

Different from the abovementioned works, we propose a long-term cross-layer joint optimization of rate control and power allocation scheme for D2D relay networks by combining Lyapunov optimization and ADMM. Various constraints of network reliability, transmission delay, and power consumption have been taken into consideration. The difference between [10] and our work is that instead of solving the large-scale network optimization problem directly, we develop a low-complexity power allocation algorithm based on ADMM.

The proposed algorithm is not constrained to D2D-relay networks. It can be extended to solve similar joint rate control and power allocation problems in different application scenarios such as task offloading [8] and energy harvesting [16].

\section{Theoretical method}

\subsection{System model}

In the traditional cellular network, the QoS of some celledge devices cannot be well satisfied due to the long distance and time-varying channel gain between the devices and BS. Thus, D2D relay networks can be utilized to enhance cell coverage via a two-hop communication manner. As shown in Fig. 1, we consider a D2D-based relay network in a single cell, which consists of one BS and $M$

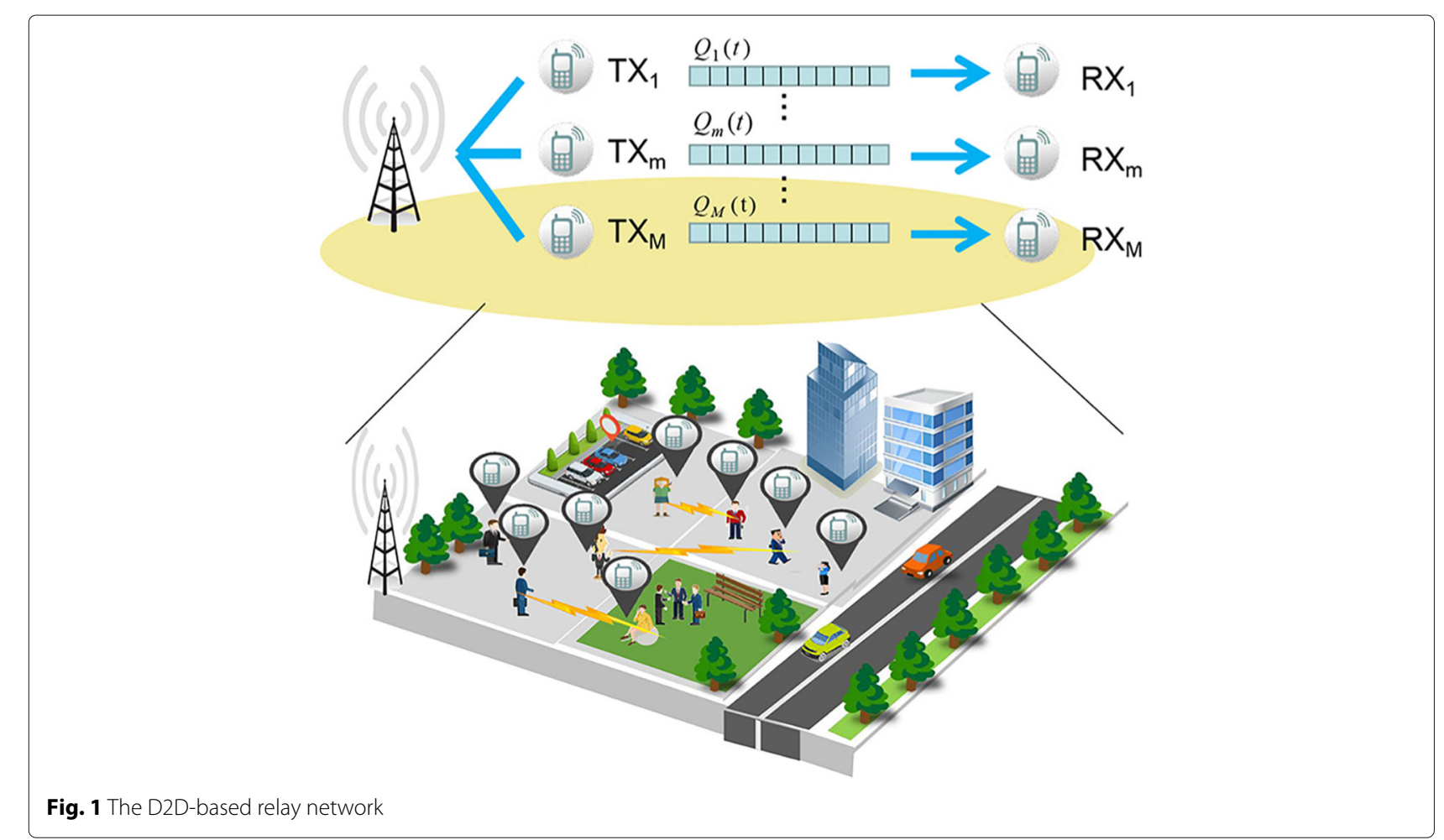


D2D pairs. In this work, we assume that the relay selection has been finished, which has been studied in many papers $[17,18]$ so that it is left out of consideration in this work. That is, there exists a one-to-one mapping between D2D TXs and D2D RXs. The BS operates in a time-slotted manner and collects the queue state information (QSI) and channel state information (CSI) at each time slot [19]. The set of time slots is defined as $\mathcal{T}=\{0, \cdots, t, \cdots, T-1\}$. Taking the D2D pair $m$ as an example, the data requested by the RX is firstly transmitted from the BS to the TX. The TX maintains a queue temporarily to store the arrival data, which is then delivered to the RX. At each time slot, assume that $A_{m}(t)$ Mbits of data arrive at the TX of D2D pair $m$ from the BS, which is assumed to be independently and identically distributed (i.i.d.) over time slots with the maximum arrival rate $A_{m, \max }$. In addition, assume that $D_{m}(t)$ Mbits of data depart from the TX of D2D pair $m$ in each time slot, which is related to the channel state and transmission power between the D2D pairs.

\subsubsection{Power allocation model}

D2D-based relay communication can be divided into inband communication (also known as LTE direct) and outband communication. In-band D2D communication can be further classified into the categories of underlay and overlay D2D communication [20]. In the circumstances of in-band overlay D2D-based relay network, the D2D communication occupies the licensed spectrum owned by the cellular operators. The cellular operators are able to employ complex interference mitigation techniques to provide higher satisfaction for D2D pairs compared to the use of unlicensed spectrum [21]. Hence, we assume that the transmission data at TXs is transmitted to the RXs through a series of orthogonal channels in the LTE direct system [22], which means there is no interference among D2D pairs. The transmission rate of D2D pair $m$ is expressed as:

$$
D_{m}(t)=B_{m}(t) \log _{2}\left(1+\frac{p_{m}(t) h_{m}^{2}(t)}{\sigma_{0}^{2}}\right),
$$

where $B_{m}(t)$ represents the channel bandwidth allocated to the $\mathrm{D} 2 \mathrm{D}$ pair $m, p_{m}(t)$ is the transmission power of $\mathrm{D} 2 \mathrm{D}$ pair $m, h_{m}(t)$ is the channel gain of D2D pair $m$, and $\sigma_{0}^{2}$ is the power of additive white Gaussian noise. Without loss of generality, assume that $h_{m}(t)$ is i.i.d. over time slots, and takes values in a finite state space. Moreover, $h_{m}(t)$ keeps constant during one time slot but varies across different time slots.

In order to reduce the power consumption of the network, the long-term time-average power consumption for arbitrary D2D pair $m$ is defined as:

$$
0 \leq \lim _{T \rightarrow \infty} \frac{1}{T} \sum_{t=0}^{T-1} p_{m}(t) \leq P_{m, a v e}
$$

and instantaneous transmission power for each time slot $t$ is defined as:

$$
0 \leq \sum_{m=1}^{M} p_{m}(t) \leq P_{\max } .
$$

where $P_{m, a v e}$ and $P_{\max }$ are the time-average and instantaneous power consumption constraints, respectively.

\subsubsection{Queue backlog model and satisfaction model}

Due to the fact that the requested data cannot be transmitted instantaneously to the RX, it has to be stored at the queue of the TX temporarily. The queue backlogs at the TXs are denoted as $\mathbf{Q}(\mathbf{t}) \triangleq\left\{Q_{1}(t), \cdots, Q_{m}(t), \cdots, Q_{M}(t)\right\}$ at each time slot $t$, which are determined by the arrival rate and the transmission rate. Hence, the dynamic queue consists of the arrival data and the departure data. The data arrival process of the queue is determined by the rate control policy, which affects the amount of data enters into the queue and the satisfaction of D2D pairs. The data departure process of the queue is determined by the power allocation policy, which affects the amount of data leaves the queue and the network latency and stability. Thus the queue $Q_{m}(t)$ at TX of D2D pair $m$ evolves in accordance with the following expression:

$$
Q_{m}(t+1)=\max \left\{Q_{m}(t)-D_{m}(t), 0\right\}+A_{m}(t) .
$$

There exists no data overflow if the long-term average transmission data of the queue is larger than or equal to the long-term average arrival data of the queue. Thus, the queue $Q_{m}(t)$ is mean rate stable [23] if

$$
\lim _{T \rightarrow \infty} \frac{\mathbb{E}\left\{\left|Q_{m}(T)\right|\right\}}{T}=0 .
$$

Equation (5) implies that the data in the stable network should be transmitted within finite delay and the stability of the network is guaranteed if the queue length is finite.

In addition, we define the satisfaction of D2D pair $m$ as a nondecreasing concave function [10, 24]:

$$
S_{m}\left(A_{m}(t)\right)=\gamma_{m} \log _{2}\left(A_{m}(t)\right),
$$

where $\gamma_{m}$ is a predefined parameter related to the service of RX in D2D pair $m$. The logarithmic function indicates that the marginal increment of the satisfaction declines gradually with $A_{m}(t)$.

\subsection{Problem formulation}

The objective of this paper is to maximize the long-term time-average satisfaction of D2D pairs. The optimization problem can be formulated as follows: 


$$
\begin{aligned}
& \text { P1: } \max _{\left\{A_{m}(t)\right\},\left\{p_{m}(t)\right\}} \lim _{T \rightarrow \infty} \frac{1}{T} \sum_{t=0}^{T-1} \mathbb{E}\left\{\sum_{m=1}^{M} S_{m}\left(A_{m}(t)\right)\right\}, \\
& \text { s.t. } C_{1}: A_{m}(t) \leq A_{m, \text { max }}, \forall m, t, \\
& C_{2}: p_{m}(t) \geq 0, \forall m, t, \\
& C_{3}: \sum_{m=1}^{M} p_{m}(t) \leq P_{\max }, \forall t, \\
& C_{4}: \lim _{T \rightarrow \infty} \frac{1}{T} \sum_{t=0}^{T-1} p_{m}(t) \leq P_{m, a v e}, \forall m, \\
& C_{5}: Q_{m}(t) \text { is mean rate stable, } \forall m, t .
\end{aligned}
$$

where $C_{1}$ represents that the arrival rate cannot exceed the maximum tolerance rate of TX. $C_{2} \sim C_{4}$ are the nonnegative transmission power, instantaneous transmission power, and time-average transmission power constraints, respectively. $C_{5}$ denotes the queue stability constraint in Eq. (5).

Next, we propose a cross-layer online optimization algorithm to solve P1 based on the Lyapunov optimization algorithm and ADMM algorithm.

\subsection{Joint rate control and power allocation optimization}

In this section, we firstly transform the long-term timeaverage optimization objective into a series of online subproblems by using the Lyapunov optimization. Then, we describe the detailed procedures of cross-layer joint optimization problem of rate control and power allocation.

\subsubsection{Problem transformation}

It is noticed that there exist long-term constraints in original problem P1. To handle the long-term time-average power consumption constraint, $C_{4}$ can be transformed into queue stability constraint by employing virtual queue [25]. The virtual queue is defined as $\mathbf{Z}(\mathbf{t}) \triangleq$ $\left\{Z_{1}(t), \cdots, Z_{m}(t), \cdots, Z_{M}(t)\right\}$, and $Z_{m}(t)$ evolves as follows:

$$
Z_{m}(t+1)=\max \left\{Z_{m}(t)-P_{m, a v e}, 0\right\}+p_{m}(t)
$$

It is worth noting that there is no actual queue data in queue $Z_{m}(t)$, which is only proposed to satisfy constraint $C_{4}$.

Theorem 1 If virtual queue $Z_{m}(t)$ is mean rate stable, then $C_{4}$ holds automatically.

Proof The detailed proof can be found in [10].
According to Theorem 1, $\mathbf{P 1}$ can be rewritten as:

$$
\begin{aligned}
& \text { P2: } \max _{\left\{A_{m}(t)\right\},\left\{p_{m}(t)\right\}} \lim _{T \rightarrow \infty} \frac{1}{T} \sum_{t=0}^{T-1} \mathbb{E}\left\{\sum_{m=1}^{M} S_{m}\left(A_{m}(t)\right)\right\}, \\
& \text { s.t. } C_{1} \sim C_{3}, \\
& C_{6}: Q_{m}(t), Z_{m}(t) \text { are mean rate stable, } \forall m, t .
\end{aligned}
$$

\subsubsection{Lyapunov optimization}

Let $\Theta(t)=[\mathbf{Q}(\mathbf{t}), \mathbf{Z}(\mathbf{t})]$ be the concatenated vector of queue length in the network. Define the Lyapunov function as a measure of total queue length at each time slot $t$ :

$$
L(\Theta(t))=\frac{1}{2} \sum_{m=1}^{M}\left\{Q_{m}^{2}(t)+Z_{m}^{2}(t)\right\},
$$

At each time slot, the conditional Lyapunov drift is expressed as:

$$
\Delta(\Theta(t)) \triangleq \mathbb{E}\{L(\Theta(t+1))-L(\Theta(t)) \mid \Theta(t)\}
$$

At each time slot, it can be observed that the Lyapunov function is able to control Lyapunov drift's ultimate value by adjusting the final queue length. According to the Little's Theorem [26], the average delay is proportional to the average queue length, which is expressed as:

$$
D_{\text {net }}=\frac{\lim _{T \rightarrow \infty} \frac{1}{T} \sum_{t=0}^{T-1} \mathbb{E}\left\{Q_{m}(t)\right\}}{\lim _{T \rightarrow \infty} \frac{1}{T} \sum_{t=0}^{T-1} \mathbb{E}\left\{A_{m}(t)\right\}},
$$

where $D_{n e t}$ is the time-average delay, which can be adjusted by minimizing Lyapunov drift.

To minimize the network delay and maximize the longterm time-average satisfaction of all D2D pairs, the driftminus-reward term is defined as:

$$
\Delta(\Theta(t))-V \mathbb{E}\left\{\sum_{m=1}^{M} S_{m}\left(A_{m}(t)\right) \mid \Theta(t)\right\},
$$

where $V$ is a non-negative control parameter that is chosen to affect the relative performance of the network delay and satisfaction of D2D pairs, i.e., the tradeoff between "network delay minimization" and "satisfaction maximization of D2D pairs".

Theorem 2 At each time slot, under any possible $\Theta(t)$ with given $V \geq 0$, the drift-minus-reward term is upper bounded by: 


$$
\begin{aligned}
& \Delta(\Theta(t))-V \mathbb{E}\left\{\sum_{m=1}^{M} S_{m}\left(A_{m}(t)\right) \mid \Theta(t)\right\} \\
& \leq C+\sum_{m=1}^{M} \mathbb{E}\left\{Q_{m}(t) A_{m}(t)-V S_{m}\left(A_{m}(t)\right) \mid \Theta(t)\right\} \\
& +\sum_{m=1}^{M} \mathbb{E}\left\{Z_{m}(t)\left(p_{m}(t)-P_{m, a v e}\right) \mid \Theta(t)\right\} \\
& -\sum_{m=1}^{M} \mathbb{E}\left\{Q_{m}(t) D_{m}(t) \mid \Theta(t)\right\}
\end{aligned}
$$

where $C$ is a positive constant which satisfies:

$$
\begin{aligned}
C & \geq \frac{1}{2} \sum_{m=1}^{M} \mathbb{E}\left\{A_{m}^{2}(t)+D_{m}^{2}(t) \mid \Theta(t)\right\} \\
& +\frac{1}{2} \sum_{m=1}^{M} \mathbb{E}\left\{p_{m}^{2}(t)+\left(P_{m, a v e}\right)^{2} \mid \Theta(t)\right\} .
\end{aligned}
$$

Proof The detailed proof can be found in Appendix 1.

According to the principle of Lyapunov optimization, P2 can be transformed into optimizing the drift-minusreward with the constraints $C_{1} \sim C_{3}$. The second term of the right hand side (RHS) in (14) involves only the rate control variables $\left\{A_{m}(t)\right\}$, while the third and the fourth term of the RHS in (14) involves only the power allocation variables $\left\{p_{m}(t)\right\}$. Therefore, $\mathbf{P 2}$ can be decoupled into two independent rate control subproblem and power allocation subproblem.

\subsubsection{Rate control}

The rate control subproblem can be formulated as:

$$
\begin{aligned}
& \text { P3 }: \min _{\left\{A_{m}(t)\right\}} \sum_{m=1}^{M}\left\{Q_{m}(t) A_{m}(t)-V S_{m}\left(A_{m}(t)\right)\right\} \\
& \text { s.t. } C_{1}
\end{aligned}
$$

The second-order derivative of $\mathbf{P 3}$ is greater than zero, which indicates that it is a convex function with respect to $\left\{A_{m}(t)\right\}$ and can be solved by KKT conditions [27]. The Lagrangian associated with $Q_{m}(t) A_{m}(t)-V S_{m}\left(A_{m}(t)\right)$ is expressed as:

$$
\begin{aligned}
& L\left(A_{m}(t), \lambda\right) \\
& =Q_{m}(t) A_{m}(t)-V S_{m}\left(A_{m}(t)\right)+\lambda\left(A_{m}(t)-A_{m, \text { max }}\right),
\end{aligned}
$$

where $\lambda$ is the Lagrange multiplier.

The first-order conditions of (17) with respect to $A_{m}(t)$ is expressed as:

$$
\frac{\partial L}{\partial A_{m}(t)}=Q_{m}(t)-\frac{V \gamma_{m}}{A_{m}(t) \ln 2}+\lambda=0,
$$

Considering the primal constraint $A_{m}(t) \leq A_{m, \max }$, dual constraint $\lambda \geq 0$ and complementary slackness constraint $\lambda\left(A_{m}(t)-A_{m, \max }\right)=0$, the optimal rate is given by:

$$
A_{m}^{*}(t)=\min \left\{\frac{\gamma_{m} V}{Q_{m}(t) \ln 2}, A_{m, \max }\right\} .
$$

It is noticed that the optimal rate is inversely proportional to the queue length $Q_{m}(t)$. Thus the online algorithm can adjust the arrival rate based on the queue length.

\subsubsection{ADMM-based power allocation algorithm}

The power allocation subproblem is expressed as:

$$
\begin{aligned}
& \text { P4 : } \min _{\left\{p_{m}(t)\right\}}\left\{\sum_{m=1}^{M} Z_{m}(t)\left(p_{m}(t)-P_{m, a v e}\right)\right. \\
& \left.-Q_{m}(t) D_{m}(t)\right\}
\end{aligned}
$$$$
\text { s.t. } C_{2}, C_{3} \text {. }
$$

It can be proved that $\mathbf{P 4}$ is a convex function regarding to $p_{m}(t)$ by calculating the corresponding second-order derivative. However, the calculating time by using toolbox to solve the optimization objective is very large due to the coupled power variables and the dynamically increasing of D2D pairs. Hence, we develop a low complexity ADMM-based power allocation algorithm to solve the problem P4.

The ADMM algorithm is simple but powerful to research distributed convex optimization problems, which had been successfully applied in many aspects, i.e., statistical learning problems, time-series analysis and scheduling [28]. In general, the diverse application domains are characterized with the large-scale problems, high-dimensional data processing and distributed collection of large scale data in stochastic process [29]. The basic produces of ADMM algorithm is to alternatively update primal variables and dual variables in an iterative manner [12]. It is beneficial to find the optimal solution with low computational complexity by using decompositioncoordination procedure.

In order to obtain the optimal solution, we rewrite the power variables into $\mathbf{p} \mathbf{1}=\left\{p_{1}, p_{2}, \cdots, p_{n}\right\}$ and $\mathbf{p} \mathbf{2}=$ $\left\{p_{n+1}, p_{n+2}, \cdots, p_{M}\right\}$. Then let $\mathbf{x}=\mathbf{p} \mathbf{1}$ and $\mathbf{z}=\mathbf{p} 2$. Therefore, we can transform $\mathbf{P 4}$ into the following ADMM format [30]:

$$
\begin{aligned}
& \mathbf{P 5}: \min _{\mathbf{x}, \mathbf{z}} f(\mathbf{x})+g(\mathbf{z}) \\
& \text { s.t. } C_{7}: \mathbf{J x}+\mathbf{K z} \leq c,
\end{aligned}
$$


where $\mathbf{x} \in \mathbf{R}^{n \times 1}, \mathbf{z} \in \mathbf{R}^{(M-n) \times 1}, \mathbf{J} \in \mathbf{R}^{1 \times n}, \mathbf{K} \in \mathbf{R}^{1 \times(M-n)}$, and $c=P_{\max }$. $\mathbf{J}$ and $\mathbf{K}$ are unit vectors. $f(\mathbf{x})$ and $g(\mathbf{z})$ satisfy:

$$
\begin{aligned}
f(\mathbf{x}) & =\sum_{m=1}^{n} f\left(x_{m}\right)=\sum_{m=1}^{n}\left\{Z_{m}(t)\left(p_{m}(t)-P_{m, \text { ave }}\right)\right. \\
& \left.-Q_{m}(t) \log _{2}\left(1+\frac{p_{m}(t) h_{m}^{2}(t)}{\sigma_{0}^{2}}\right)\right\} \\
g(\mathbf{z}) & =\sum_{m=n+1}^{M} g\left(z_{m}\right)=\sum_{m=n+1}^{M}\left\{Z _ { m } ( t ) \left(p_{m}(t)\right.\right. \\
& \left.\left.-P_{m, a v e}\right)-Q_{m}(t) \log _{2}\left(1+\frac{p_{m}(t) h_{m}^{2}(t)}{\sigma_{0}^{2}}\right)\right\}
\end{aligned}
$$

There exist two basic forms of the ADMM algorithm, such as the unscaled form and the scaled form [16]. For the sake of simplicity, the scaled ADMM algorithm is employed in this paper. The augmented Lagrangian of P5 is expressed as:

$$
\begin{aligned}
& L_{\rho}(\mathbf{x}, \mathbf{z}, \boldsymbol{\beta})=f(\mathbf{x})+g(\mathbf{z}) \\
& +\frac{\boldsymbol{\rho}}{2}\left\|\mathbf{J} \mathbf{x}+\mathbf{K z}-c+\frac{1}{\boldsymbol{\rho}} \boldsymbol{\beta}\right\|_{2}^{2}-\frac{\boldsymbol{\rho}}{2}\left\|\frac{1}{\boldsymbol{\rho}} \boldsymbol{\beta}\right\|_{2}^{2} .
\end{aligned}
$$

where $\rho>0$ represents the penalty parameter, which is related to the convergence speed of ADMM algorithm. $\boldsymbol{\beta}$ is the vector form of the Lagrange multipliers.

The ADMM algorithm in scaled form consists of the following iterations regarding to the primal variables and Lagrange multipliers:

$$
\begin{aligned}
& \mathbf{x}[i+1]:=\arg \min _{\mathbf{x}}\left\{f(\mathbf{x})+\frac{\rho}{2} \| \mathbf{J} \mathbf{x}+\mathbf{K z}[i]-c\right. \\
&\left.+\frac{1}{\boldsymbol{\rho}} \boldsymbol{\beta}[i] \|_{2}^{2}\right\}, \\
& \mathbf{z}[i+1]:=\arg \min _{\mathbf{z}}\left\{g(\mathbf{z})+\frac{\boldsymbol{\rho}}{2} \| \mathbf{J x}[i+1]+\mathbf{K} \mathbf{z}-c\right. \\
&\left.+\frac{1}{\boldsymbol{\rho}} \boldsymbol{\beta}[i] \|_{2}^{2}\right\}, \\
& \boldsymbol{\beta}[i+1]:=\boldsymbol{\beta}[i]+\boldsymbol{\rho}(\mathbf{J x}[i+1]-\mathbf{K z}[i+1]-c) .
\end{aligned}
$$

where $i$ denotes the index of iteration.

Next, based on the analysis of optimality conditions [31], the primal residual is expressed as:

$$
\mathbf{r}[i+1]=\mathbf{J x}[i+1]+\mathbf{K} \mathbf{z}[i+1]-c,
$$

and the dual residual is expressed as:

$$
\mathbf{s}[i+1]=\rho \mathbf{J}^{T} \mathbf{K}(\mathbf{z}[i+1]-\mathbf{z}[i]) .
$$

Therefore, the reasonable termination criteria satisfies:

$$
\|\mathbf{r}[i]\|_{2} \leq \epsilon^{\text {pri }} \text { and }\|\mathbf{s}[i]\|_{2} \leq \epsilon^{\text {dual }} \text {. }
$$

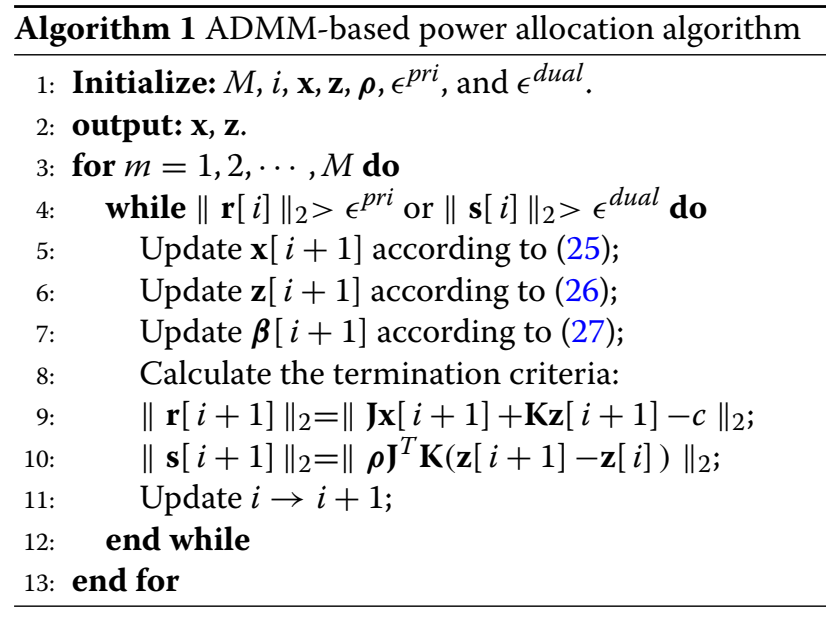

where $\epsilon^{p r i}>0$ and $\epsilon^{\text {dual }}>0$ denote feasibility tolerances with respect to primal conditions and dual conditions. Consequently, the ADMM-based power allocation algorithm is summarized in Algorithm 1.

\subsection{Performance analysis}

In this subsection, we analyze the performance of Lyapunov optimization algorithm and ADMM algorithm, respectively.

\subsubsection{Performance of Lyapunov optimization algorithm}

Due to the fact that all physical quantities cannot be infinitely large in the practical network, we consider that the arrival rate, transmission rate, power consumption, and satisfaction of $\mathrm{D} 2 \mathrm{D}$ pairs are all bounded, i.e., $\mathbb{E}\left\{A_{m}(t)\right\} \leq \theta, \mathbb{E}\left\{D_{m}(t)\right\} \leq \theta, \mathbb{E}\left\{p_{m}(t)\right\} \leq \theta, S_{\text {min }} \leq$ $\mathbb{E}\left\{S_{m}\left(A_{m}(t)\right)\right\} \leq S_{\text {max }}$, where $\theta, S_{\text {min }}$ and $S_{\text {max }}$ are finite non-negative constants.

Assume that there is at least one feasible solution to problem P1 which satisfies constraints $C_{1} \sim C_{5}$ and the bounded values mentioned above. For arbitrary small positive real number $\varepsilon$ and $\zeta$, the following expressions hold [32]:

$$
\begin{aligned}
& \mathbb{E}\left\{A_{m}^{*}(t)-D_{m}^{*}(t) \mid \Theta(t)\right\}=\mathbb{E}\left\{A_{m}^{*}(t)-D_{m}^{*}(t)\right\} \leq-\varepsilon \\
& \mathbb{E}\left\{p_{m}^{*}(t)-P_{m, \text { ave }} \mid \Theta(t)\right\}=\mathbb{E}\left\{p_{m}^{*}(t)-P_{m, \text { ave }}\right\} \leq-\zeta \\
& \mathbb{E}\left\{S_{m}^{*}\left(A_{m}^{*}(t)\right) \mid \Theta(t)\right\}=\mathbb{E}\left\{S_{m}^{*}\left(A_{m}^{*}(t)\right)\right\}=S_{\text {opt }}
\end{aligned}
$$

where $A_{m}^{*}(t), D_{m}^{*}(t), p_{m}^{*}(t)$, and $S_{m}^{*}\left(A_{m}^{*}(t)\right)$ are the corresponding resulting values, and $S_{\text {opt }}$ denotes the theoretical optimal value.

Theorem 3 Suppose that the problem P1 is feasible, $h_{m}(t)$ is i.i.d. with slotted time, and that $\mathbb{E}\{L(\Theta(0))\}<\infty$. 
Table 1 Parameter table

\begin{tabular}{ll}
\hline Parameters & Value \\
\hline Cell radius & $300 \mathrm{~m}$ \\
D2D pairs & 4 \\
Subchannels & 4 \\
Time slot & 100 \\
$P_{\max }$ & $0.8 \mathrm{~W}$ \\
$\rho$ & 5 \\
$\sigma_{0}^{2}$ & $-114 \mathrm{dbm}$ \\
$\gamma_{1}$ to $\gamma_{4}$ & $0.1,0.2,0.3,0.4$ \\
$P_{1, \text { ave to }} P_{4, \text { ave }}$ & $0.1,0.2,0.3,0.4 \mathrm{~W}$ \\
Bandwidth $B_{1}$ to $B_{4}$ & $2,2,2,2 \mathrm{MHZ}$ \\
Control parameter $V$ & $300-700$ \\
Arrival rate $A_{\max }$ & $10 \mathrm{Mbits}$ \\
\hline
\end{tabular}

For arbitrary $V \geq 0$, the following properties corresponding to the proposed algorithm hold:

1. $Q_{m}(t)$ and $Z_{m}(t)$ are mean rate stable, which guarantee the constraint $C_{6}$.

2. The time-average satisfaction of $D 2 D$ pairs satisfies:

$$
\lim _{T \rightarrow \infty} \frac{1}{T} \sum_{t=0}^{T-1} \mathbb{E}\left\{S_{m}\left(A_{m}(t)\right)\right\} \geq S_{\text {opt }}-\frac{C}{V}
$$

3. The time-average queue length satisfies:

$$
\lim _{T \rightarrow \infty} \frac{1}{T} \sum_{t=0}^{T-1} \sum_{m=1}^{M} \mathbb{E}\left\{Q_{m}(t)\right\} \leq \frac{C+V\left(S_{\max }-S_{\text {opt }}\right)}{\varepsilon}
$$

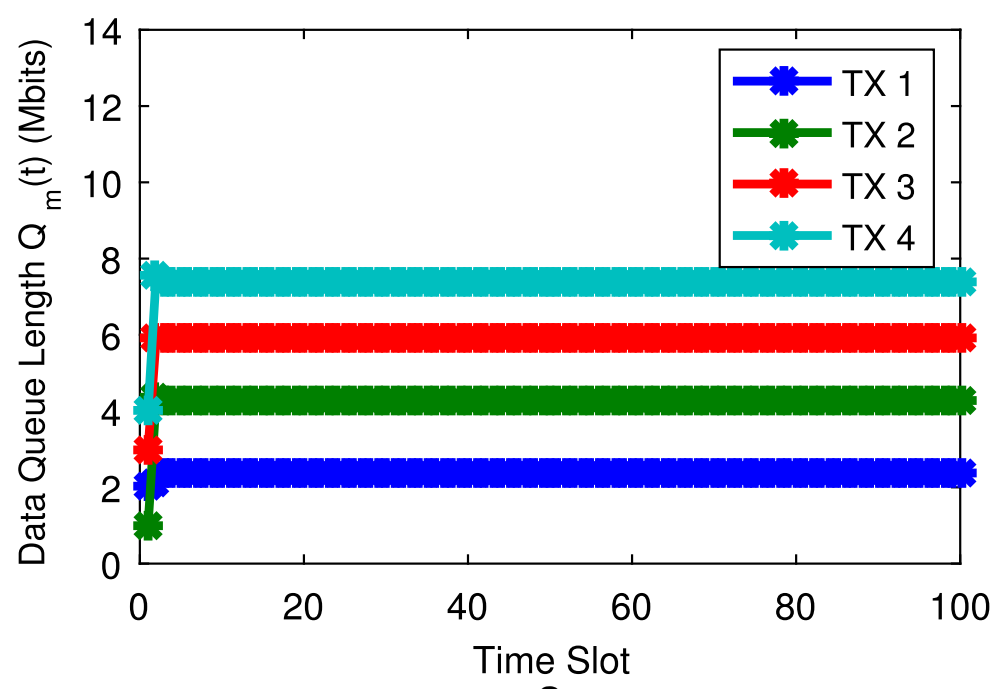

a

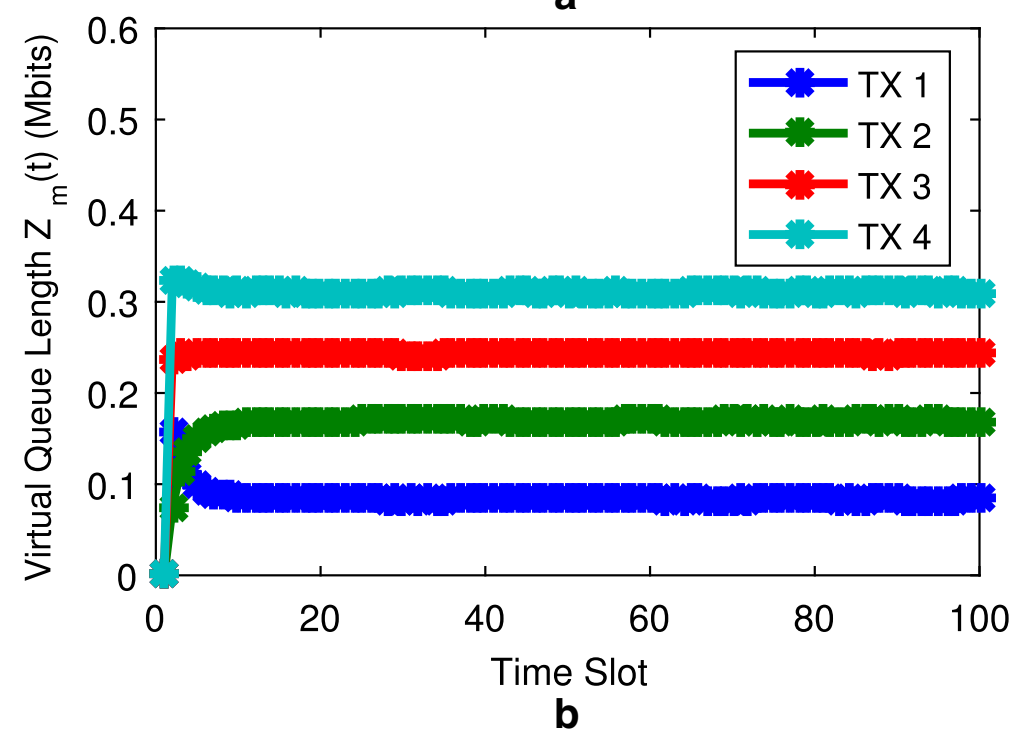

Fig. 2 a, b Queue stability versus time slots 
Proof The detailed proof can be found in Appendix 2.

Based on the above analysis, we conclude that the proposed rate control and power allocation algorithm can satisfy the queue stability constraint and achieve the trade-off between network delay and satisfaction of D2D pairs by adjusting parameter $V$.

\subsubsection{Convergence of ADMM algorithm}

The objective function of P5 is closed, proper, and convex, and the Lagrangian $L_{0}(\mathbf{x}, \mathbf{z}, \boldsymbol{\beta})$ has a saddle point. Thus, the iterations satisfy the following convergence properties.
Theorem 4 The residual convergence, objective convergence and dual variable convergence are expressed as follows:

1. Residual convergence: The primal and dual residuals converge to 0 as $i \rightarrow \infty$, which implies that the iterations approach feasibility.

2. Objective convergence: The objective function of $\mathbf{P 5}$ eventually converges to the optimal value under the stopping criterion as $i \rightarrow \infty$.

3. Dual variable convergence: The dual variable $\boldsymbol{\beta}[i+1]$ converges to dual optimal value as $i \rightarrow \infty$.

Proof The detailed proof can be found in [33].
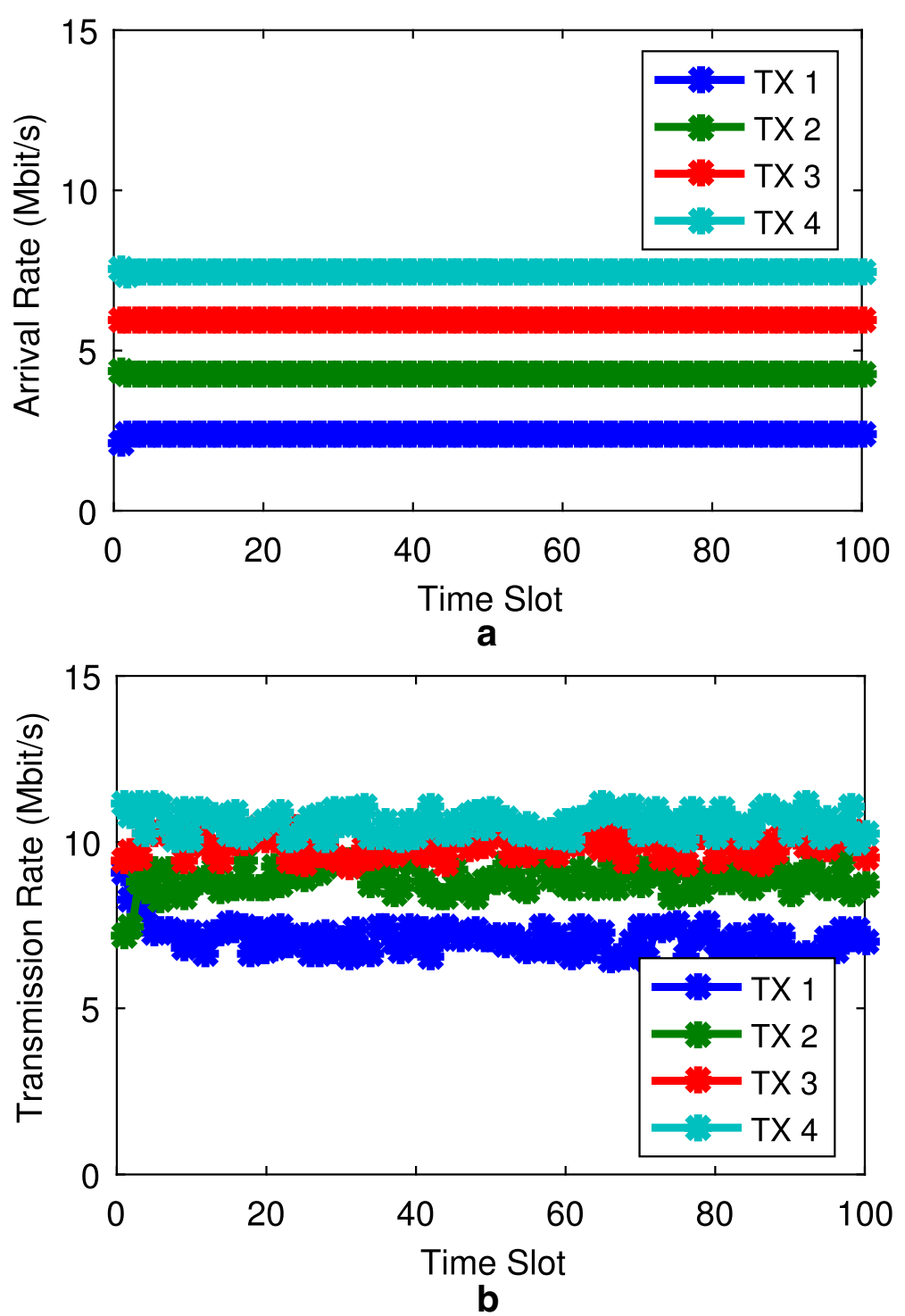

Fig. 3 a, b Rate stability versus time slots 


\section{Results and discussion}

In this section, we verify the system performance of the proposed algorithm through simulation results. Assume that there are $M=4 \mathrm{D} 2 \mathrm{D}$ pairs for data transmission in each time slot, and the corresponding number of subchannels is set as 4. Detailed parameters are summarized in Table 1 [10, 34, 35].

Figure 2 shows the queue length of the data queue $Q_{m}(t)$ and the virtual power queue $Z_{m}(t)$ versus time slots, respectively. It can be observed that both the data queue and the virtual queue are bounded after a period of time, which guarantees the stability of network. The phenomenon can be well explained by the first property in Theorem 3.

Figure 3 shows the transmission rate and arrival rate versus time slots, respectively. It can be observed that both the transmission rate and arrival rate are stable, which guarantees the long-term time-averaged power constraint. In addition, the fluctuation of arrival rate in Fig. $3 \mathrm{a}$ is smaller than that of transmission rate in Fig. 3b. The reason is that the arrival rate is only related to the stable queues $\mathbf{Q}(\mathbf{t})$ and $\mathbf{Z}(\mathbf{t})$, while the transmission rate is not only related to the stable queues $\mathbf{Q}(\mathbf{t})$ and $\mathbf{Z}(\mathbf{t})$, but also related to the transmission power and channel gain, which is varying across time slots. Therefore, the transmission rate remains stable over a larger range of values compared with the arrival rate.

Figure 4 shows the satisfaction of D2D pairs and the average network delay versus the control parameter $V$, respectively. It can be observed that the satisfaction of D2D pairs and the average network delay increase as $V$ increases. The reason is consistent with the second and third properties of Theorem 3. Furthermore,
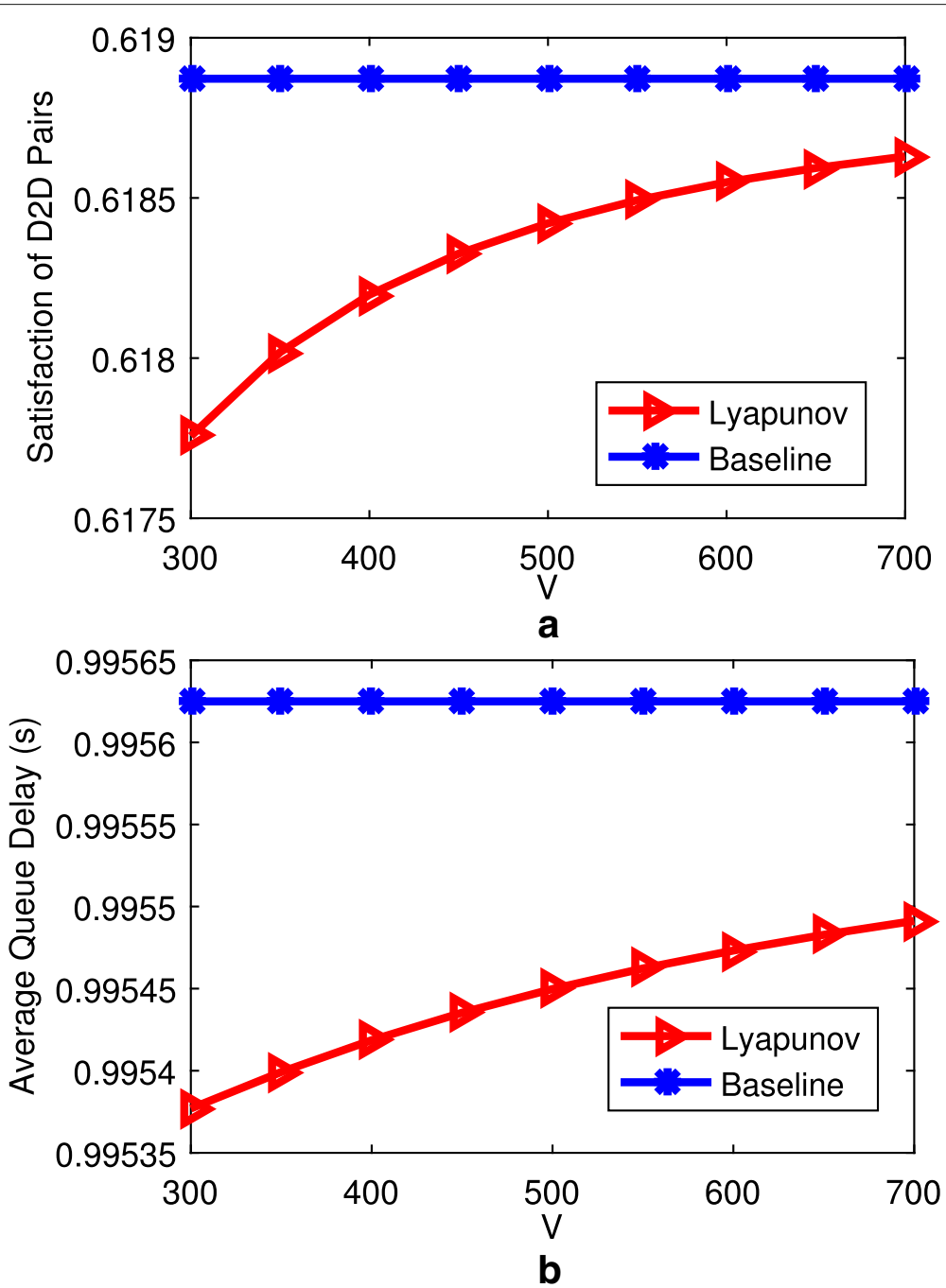

Fig. 4 a, b Satisfaction of D2D pairs and network delay versus control parameter $V$ 

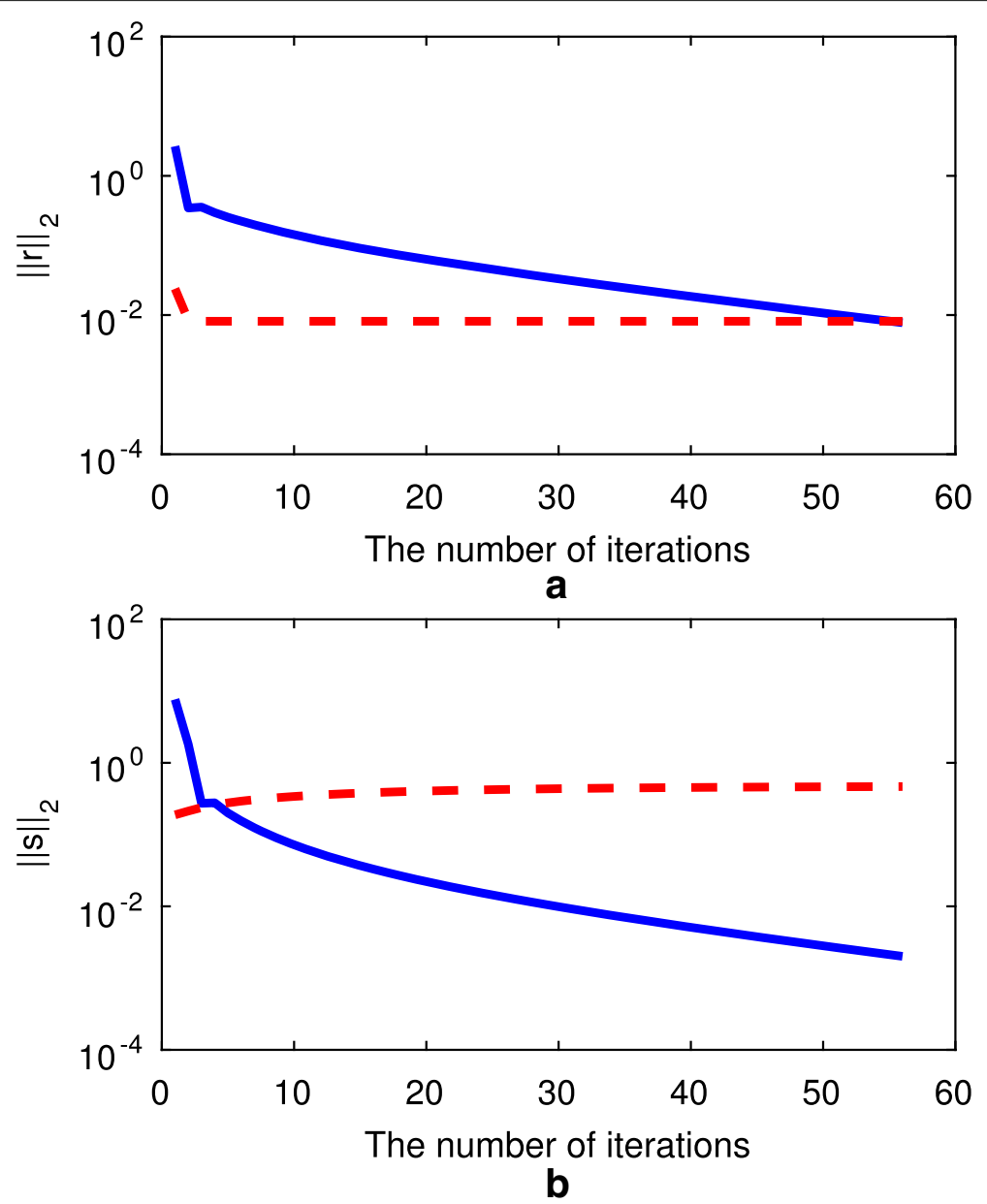

Fig. $\mathbf{5} \mathbf{a}, \mathbf{b}$ The residual convergence versus the number of iterations

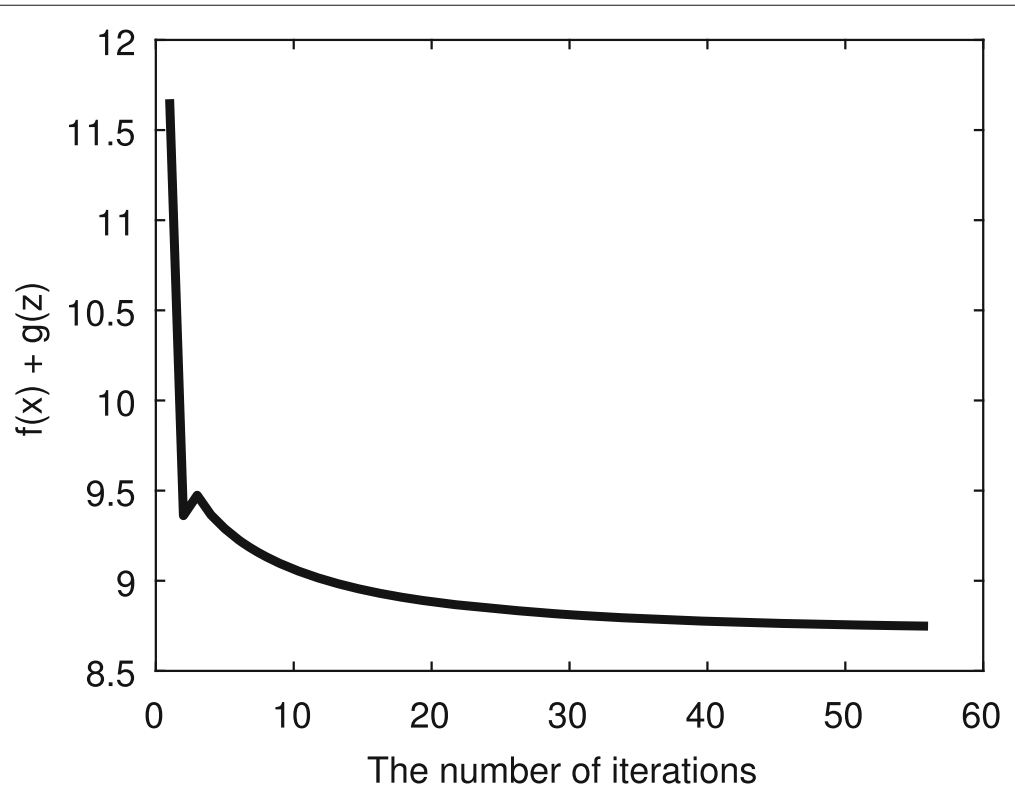

Fig. 6 The objective convergence versus the number of iterations 
the snapshot based algorithm [10] is denoted as the baseline algorithm to maximize instantaneous satisfaction of all D2D pairs for the purpose of comparison. Typically, the baseline algorithm only considers the service demands of users in short-term while ignoring the power constraint and stability of the network in longterm. It can be observed that the proposed algorithm can approximate to $99.9 \%$ of the optimal satisfaction of D2D pairs with lower average network delay. The reason is that the proposed algorithm can simultaneously optimize the arrival rate in network layer and the power allocation in physical layer from a global long-term perspective. Figure 5 shows the residual convergence of the ADMM algorithm versus the number of iterations. The stopping criterion constraints $\epsilon^{p r i}$ and $\epsilon^{\text {dual }}$ are represented by the dotted lines in Fig. $5 \mathrm{a}$ and b, respectively. It can be observed that the stopping criterion can be satisfied after 57 iterations in Fig. 5a and 4 iterations in Fig. 5b. The iterations will stop if and only if both the primal residual and the dual residual conditions are satisfied simultaneously, i.e., after 57 iterations, which is consistent with the first property of Theorem 4.

Figure 6 shows the the optimal convergence of the ADMM algorithm versus the number of iterations. It shows the convergence of objective function, which demonstrates that the proposed algorithm can obtain the optimal solution of the objective function, i.e., 8.75. The reason can be explained by the second property of Theorem 4 . In addition, the dual variables will converge after multiple iterations due to the derivation from the primal variables, which is consistent with the third property of Theorem 4.

Table 2 shows the simulation analysis by using CVX toolbox approach and ADMM-based power allocation algorithm. On one hand, it can be observed that the convergence time of ADMM-based power allocation algorithm requires 0.021236 seconds, which is only about $1.7 \%$ of the convergence time by using the CVX toolbox approach. Due to the fast convergence of the ADMM algorithm, the ADMM-based power allocation algorithm can get the optimal solution with lower computational complexity within error tolerance at each time slot. On the other hand, 4 TXs share the

Table 2 Comparison of algorithm complexity

\begin{tabular}{lll}
\hline 1 Term & CVX toolbox & ADMM algorithm \\
\hline Calculating time & $1.232408 \mathrm{~s}$ & $0.021236 \mathrm{~s}$ \\
Power allocation & $p_{1}=0.090546$ & $p_{1}=0.082289$ \\
& $p_{2}=0.171437$ & $p_{2}=0.168075$ \\
& $p_{3}=0.237371$ & $p_{3}=0.241891$ \\
& $p_{4}=0.300646$ & $p_{4}=0.315196$
\end{tabular}

total power of $0.8 \mathrm{~W}$ at each time slot, which proves the feasibility of the proposed power allocation algorithm. The table shows that the power allocation varied with the channel states and the service demands of different TXs.

\section{Conclusion}

In this paper, we propose the D2D-based relay framework to improve the reliability and reduce delay of the network. Based on the Lyapunov optimization, the crosslayer joint optimization problem is separated into two independent rate control subproblem and power allocation subproblem, which can be solved by using KKT conditions and ADMM algorithm, respectively. The tradeoff between network delay minimization and satisfaction maximization of D2D pairs with $\left[\mathcal{O}\left(\frac{1}{V}\right), \mathcal{O}(V)\right]$ can be obtained by the proposed algorithm, which has been verified by the simulation results.

In the future work, we will improve the performance of the network while considering device mobility from a long-term perspective. Furthermore, we will study how to combine Lyapunov optimization and ADMM algorithm with big data or machine learning to deal with high complexity problems.

\section{Appendix}

\section{Appendix 1: Proof of Theorem 2}

According to $(\max \{Q-b, 0\}+A)^{2} \leq Q^{2}+b^{2}+A^{2}+2 Q(A-$ $b$ ), where $\mathrm{Q}, \mathrm{b}$ and $\mathrm{A}$ are non-negative real numbers, we obtain the following expression:

$$
\begin{aligned}
\Delta(\Theta(t)) & \triangleq \mathbb{E}\{L(\Theta(t+1))-L(\Theta(t)) \mid \Theta(t)\} \\
& \leq C+\sum_{m=1}^{M} \mathbb{E}\left\{Q_{m}(t)\left[A_{m}(t)-D_{m}(t)\right] \mid \Theta(t)\right\} \\
& +\sum_{m=1}^{M} \mathbb{E}\left\{Z_{m}(t)\left(p_{m}(t)-P_{m, \text { ave }}\right) \mid \Theta(t)\right\}
\end{aligned}
$$

where

$$
\begin{aligned}
C & \geq \frac{1}{2} \sum_{m=1}^{M} \mathbb{E}\left\{A_{m}^{2}(t)+D_{m}^{2}(t) \mid \Theta(t)\right\} \\
& +\frac{1}{2} \sum_{m=1}^{M} \mathbb{E}\left\{p_{m}^{2}(t)+\left(P_{m, \text { ave }}\right)^{2} \mid \Theta(t)\right\} .
\end{aligned}
$$

Add and subtract $V \mathbb{E}\left\{\sum_{m=1}^{M} S_{m}\left(A_{m}(t)\right) \mid \Theta(t)\right\}$ to the both sides of Eq. (36), then merge the same variables to prove (14). 


\section{Appendix 1: Proof of Theorem 3}

In order to minimize the RHS of (14) with constraints $C_{1} \sim C_{3}$, we obtain the following expression:

$$
\begin{aligned}
& \Delta(\Theta(t))-V \mathbb{E}\left\{\sum_{m=1}^{M} S_{m}\left(A_{m}(t)\right) \mid \Theta(t)\right\} \\
& \leq C+\sum_{m=1}^{M} \mathbb{E}\left\{Q_{m}(t)\left[A_{m}^{*}(t)-D_{m}^{*}(t)\right] \mid \Theta(t)\right\} \\
& +\sum_{m=1}^{M} \mathbb{E}\left\{Z_{m}(t)\left(p_{m}^{*}(t)-P_{m, a v e}\right) \mid \Theta(t)\right\}, \\
& -V \mathbb{E}\left\{\sum_{m=1}^{M} S_{m}^{*}\left(A_{m}^{*}(t)\right) \mid \Theta(t)\right\},
\end{aligned}
$$

Plugging (31), (32) and (33) into (38), and taking $\zeta \rightarrow 0$, the following expression satisfys:

$$
\begin{aligned}
& \Delta(\Theta(t))-V \mathbb{E}\left\{\sum_{m=1}^{M} S_{m}\left(A_{m}(t)\right) \mid \Theta(t)\right\} \\
& \quad \leq C-V \mathbb{E}\left\{S_{o p t} \mid \Theta(t)\right\}-\varepsilon \sum_{m=1}^{M} \mathbb{E}\left\{Q_{m}(t) \mid \Theta(t)\right\},
\end{aligned}
$$

By applying the rules of iterated expectation and telescoping sums, we obtain:

$$
\begin{aligned}
& \mathbb{E}\{L(\Theta(t))\}-\mathbb{E}\{L(\Theta(0))\}-V \sum_{t=0}^{T-1} \mathbb{E}\left\{S_{m}\left(A_{m}(t)\right)\right\} \\
& \leq T\left(C-V S_{\text {opt }}\right)-\varepsilon \sum_{t=0}^{T-1} \sum_{m=1}^{M} \mathbb{E}\left\{Q_{m}(t)\right\},
\end{aligned}
$$

Since $\mathbb{E}\{L(\Theta(0))\}=0, \mathbb{E}\{L(\Theta(t))\} \geq 0$ and $Q_{m}(t) \geq 0$, rearrange terms and we obtain:

$$
V \sum_{t=0}^{T-1} \mathbb{E}\left\{S_{m}\left(A_{m}(t)\right)\right\} \geq T V S_{o p t}-T C
$$

Dividing by $V T$ at both sides and taking $T \rightarrow \infty$, (34) can be proved.

Similarly, by rearranging terms, (40) can be rewritten as:

$$
\begin{aligned}
\varepsilon \sum_{t=0}^{T-1} \sum_{m=1}^{M} \mathbb{E}\left\{Q_{m}(t)\right\} & \leq T\left(C-V S_{\text {opt }}\right) \\
& +V \sum_{t=0}^{T-1} \mathbb{E}\left\{S_{m}\left(A_{m}(t)\right)\right\} \\
& \leq T\left(C-V S_{\text {opt }}\right)+T V S_{\text {max }}
\end{aligned}
$$

Dividing by $\varepsilon T$ at both sides and taking $T \rightarrow \infty$, (35) can be proved.
According to the definition of Lyapunov function, taking expectation and rearranging terms yield:

$$
\sum_{m=1}^{M} \mathbb{E}\left\{Q_{m}^{2}(t)\right\}=2 \mathbb{E}\{L(\Theta(t))\}-\sum_{m=1}^{M} \mathbb{E}\left\{Z_{m}^{2}(t)\right\},
$$

We obtain the following expression by plugging (43) into (40) and rearranging terms:

$$
\sum_{m=1}^{M} \mathbb{E}\left\{Q_{m}^{2}(t)\right\} \leq 2 T V\left(S_{\text {max }}-S_{\text {opt }}\right)+T C,
$$

According to inequality $\mathbb{E}^{2}\left\{\left|Q_{m}(t)\right|\right\} \leq \mathbb{E}\left\{Q_{m}^{2}(t)\right\}$, we obtain:

$$
\begin{aligned}
\sum_{m=1}^{M} \mathbb{E}\left\{\left|Q_{m}(t)\right|\right\} & \leq \sqrt{\sum_{m=1}^{M} \mathbb{E}\left\{Q_{m}^{2}(t)\right\}} \\
& \leq \sqrt{2 T V\left(S_{\text {max }}-S_{\text {opt }}\right)+T C}
\end{aligned}
$$

Dividing by $T$ and taking $T \rightarrow \infty$, we can obtain:

$$
\lim _{T \rightarrow \infty} \frac{\sum_{m=1}^{M} \mathbb{E}\left\{\left|Q_{m}(T)\right|\right\}}{T}=0 .
$$

which proves the stability of queues by changing the order of taking expectation and limit. Queue $Q_{m}(t)$ is mean rate stable according to (5), thus $C_{5}$ can be satisfied. Similar proof can be applied to $Z_{m}(t)$ with slight modification.

\section{Abbreviations}

ADMM : Alternating direction method of multipliers; BS : Base station; CSI : Channel state information; D2D : Device-to-device; i.i.d. : Independent and identical distribution; KKT : Karush-Kuhn-Tucher; QoE : Quality of experience; QoS : Quality of service; QSI : Queue state information; RHS : Right side hand; $\mathrm{RX}$ : D2D receiver; $\mathrm{TX}$ : $\mathrm{D} 2 \mathrm{D}$ transmitter

\section{Acknowledgements}

Thanks to Professor Liangrui Tang of North China Electric Power University for his guidance of this research.

\section{Funding}

This work was partially supported by the National Natural Science Foundation of China (NSFC) under grant number 61601181; the Fundamental Research Funds for the Central Universities under grant number 2017 MS001.

\section{Availability of data and materials}

Not applicable.

\section{Authors' contributions}

YW wrote the manuscript and made a part of simulations. YH gave some suggestions and made a part of simulations. ZZ proposed the idea and revised this paper. All authors took an active role in the writing process of the document, and read and approved the final manuscript.

\section{Competing interests}

The authors declare that they have no competing interests.

\section{Publisher's Note}

Springer Nature remains neutral with regard to jurisdictional claims in published maps and institutional affiliations.

\section{Author details}

${ }^{1}$ The State Key Laboratory of Alternate Electrical Power System with Renewable Energy Sources, School of Electrical and Electronic Engineering, 
North China Electric Power University, Beijing, China. ${ }^{2}$ The Institute of Telecommunications, 3810-193 Aveiro, Portugal. ${ }^{3}$ The University of South Wales, Pontypridd CF37 1DL, UK. ${ }^{4}$ SCC, Lancaster University, Pontypridd,UK.

Received: 8 December 2018 Accepted: 28 March 2019

Published online: 02 May 2019

\section{References}

1. Z. Zhou, H. Liao, B. Gu, K. M. S. Huq, S. Mumtaz, J. Rodriguez, Robust mobile crowd sensing: when deep learning meets edge computing. IEEE Netw. 32(4), 54-60 (2018)

2. Z. Zhou, H. Yu, C. Xu, Y. Zhang, S. Mumtaz, J. Rodriguez, Dependable content distribution in D2D-based cooperative vehicular networks: a big data-integrated coalition game approach. IEEE Trans. Ind. Informat. 19(3), 953-964 (2018)

3. M. Sheng, Y. Li, X. Wang, J. Li, Y. Shi, Energy efficiency and delay tradeoff in device-to-device communications underlaying cellular networks. IEEE J. Sel. Areas Commun. 34(1), 92-106 (2016)

4. C. Xu, J. Feng, Z. Zhou, J. Wu, C. Perera, Cross-layer optimization for cooperative content distribution in multihop device-to-device networks. IEEE Internet Things J. 99, 1-1 (2017)

5. Z. Zhou, M. Dong, K. Ota, G. Wang, L. T. Yang, Energy-efficient matching for resource allocation in D2D enabled cellular networks. IEEE Internet Things J. 3(3), 428-438 (2016)

6. S. Dang, G. Chen, J. P. Coon, Multicarrier relay selection for full-duplex relay-assisted OFDM D2D systems. IEEE Trans. Veh. Technol. 67(8), 7204-7218 (2018)

7. L. Pu, X. Chen, J. Xu, X. Fu, D2D fogging: An energy-efficient and incentive-aware task offloading framework via network-assisted D2D collaboration. IEEE J. Sel. Areas Commun. 34(12), 3887-3901 (2016)

8. L. Chen, S. Zhou, J. Xu, Computation peer offloading for energy-constrained mobile edge computing in small-cell networks. IEEE/ACM Trans. Netw. 26(4), 1619-1632 (2018)

9. Y. Guo, Q. Yang, J. Liu, K. S. Kwak, Cross-layer rate control and resource allocation in spectrum-sharing OFDMA small-cell networks with delay constraints. IEEE Trans. Veh. Technol. 66(5), 4133-4147 (2017)

10. Y. Guo, Q. Yang, K. S. Kwak, Quality-oriented rate control and resource allocation in time-varying OFDMA networks. IEEE Trans. Veh. Technol. 66(3), 2324-2338 (2017)

11. M. Peng, Y. Yu, H. Xiang, H. V. Poor, Energy-efficient resource allocation optimization for multimedia heterogeneous cloud radio access networks. IEEE Trans. Multimedia. 18(5), 879-892 (2016)

12. C. Liang, F. R. Yu, H. Yao, Z. Han, Virtual resource allocation in information-centric wireless networks with virtualization. IEEE Trans. Veh. Technol. 65(12), 9902-9914 (2016)

13. N. Li, Z. Fei, C. Xing, D. Zhu, M. Lei, Robust low-complexity MMSE precoding algorithm for cloud radio access networks. IEEE Commun. Lett. 18(5), 773-776 (2014)

14. Q. Ling, Y. Liu, W. Shi, Z. Tian, Weighted ADMM for fast decentralized network optimization. IEEE Trans. Signal Process. 64(22), 5930-5942 (2016)

15. E. Chen, M. Tao, ADMM-based fast algorithm for multi-group multicast beamforming in large-scale wireless systems. IEEE Trans. Commun. 65(6), 2685-2698 (2016)

16. G. Zhang, Y. Chen, Z. Shen, L. Wang, Distributed energy management for multi-user mobile-edge computing systems with energy harvesting devices and QoS constraints. IEEE Internet Things J., 1-1 (2018)

17. Z. Zhou, F. Xiong, C. Xu, Y. He, S. Mumtaz, Energy-efficient vehicular heterogeneous networks for green cities. IEEE Trans. Ind. Informat. 14(4), 1522-1531 (2018)

18. C. Xu, J. Feng, B. Huang, Z. Zhou, S. Mumtaz, J. Rodriguez, Joint relay selection and resource allocation for energy-efficient D2D cooperative communications using matching theory. Appl. Sci. 7(5), 491-515 (2017)

19. J. Liu, Q. Yang, S. G, Congestion avoidance and load balancing in content placement and request redirection for mobile CDN. IEEE/ACM Trans. Netw. 26(2), 851-863 (2018)

20. A. Asadi, Q. Wang, V. Mancuso, A survey on device-to-device communication in cellular networks. Commun. Surveys Tuts. 16(4), 1801-1819 (2014)

21. H. Kim, J. Na, E. Cho, in Proceedings of the International Conference on Information Networking 2014. Resource allocation policy to avoid interference between cellular and D2D links/ and D2D links in mobile networks (IEEE, Phuket, 2014), pp. 588-591
22. P. Mach, Z. Becvar, T. Vanek, In-band device-to-device communication in OFDMA cellular networks: A survey and challenges. Commun. Surveys Tuts. 17(4), 1885-1922 (2015)

23. M. J. Neely, Stochastic Network Optimization with Application to Communication and Queueing Systems. (Morgan and Claypool, San Rafael, 2010)

24. M. Chen, S. S. M. Ponec, J. Li, P. A. Chou, Utility maximization in peer-to-peer systems with applications to video conferencing. IEEE/ACM Trans. Netw. 20(6), 1681-1694 (2012)

25. W. Bao, H. Chen, Y. Li, B. Vucetic, Joint rate control and power allocation for non-orthogonal multiple access systems. IEEE J. Sel. Areas Commun. 35(12), 2798-2811 (2017)

26. D. Bertsekas, R. Gallager, Data Networks. (Prentice hall, NJ,USA, 1987)

27. L. Duan, T. Kubo, K. Sugiyama, J. Huang, T. Hasegawa, J. Walrand Motivating smartphone collaboration in data acquisition and distributed computing. IEEE Trans. Mobile Comput. 13(10), 2320-2333 (2014)

28. C. Lu, J. Feng, S. Yan, Z. Lin, A unified alternating direction method of multipliers by majorization minimization. IEEE Trans. Pattern Anal. Mach. Intell. 40(3), 527-541 (2018)

29. Y. Li, G. Shi, W. Yin, L. Liu, Z. Han, A distributed ADMM approach with decomposition-coordination for mobile data offloading. IEEE Trans. Veh. Technol. 67(3), 2514-2530 (2018)

30. Y. Wang, L. Wu, S. Wang, A fully-decentralized consensus-based ADMM approach for DC-OPF with demand response. IEEE Trans. Smart Grid. 8(6), 2637-2647 (2017)

31. G. Chen, Q. Yang, An ADMM-based distributed algorithm for economic dispatch in islanded microgrids. IEEE Trans. Ind. Informat. 14(9), 3892-3903 (2018)

32. L. Georgiadis, M. J. Neely, L. Tassiulas, Resource allocation and cross-layer control in wireless networks. J. Found. Trends Netw. 1(1), 1-144 (2006)

33. S. Boyd, N. Parikh, E. Chu, B. Peleato, J. Eckstein, Distributed optimization and statistical learning via the alternating direction method of multipliers. Found. Trends Mach. Learn. 3(1), 1-122 (2011)

34. Z. Zhou, J. Feng, B. Gu, B. Ai, S. Mumtaz, J. Rodriguez, M. Guizani, When mobile crowd sensing meets $\cup A V$ : Energy-efficient task assignment and route planning. IEEE Trans. Commun. 66(11), 5526-5538 (2018)

35. Z. Zhou, K. Ota, M. Dong, C. Xu, Energy-efficient matching for resource allocation in D2D enabled cellular networks. IEEE Trans. Veh. Technol. 66(6), 5256-5268 (2017)

\section{Submit your manuscript to a SpringerOpen ${ }^{\circ}$ journal and benefit from:}

- Convenient online submission

Rigorous peer review

- Open access: articles freely available online

- High visibility within the field

- Retaining the copyright to your article

Submit your next manuscript at $\gg$ springeropen.com 